\title{
COVID-19's religious strain: Differentiating spirituality from pathology
}

Jason A. Barrett, MD

A $\mathrm{s}$ the world grapples with the coronavirus disease 2019 (COVID-19) pandemic, the search for answers, comfort, how to cope, and how to make sense of it all has become paramount. People commonly turn to their faith in times of crisis, but this recent global public health emergency is unlike many have ever seen or could have imagined. ${ }^{1}$ What happens when the well-intentioned journey for spiritual insight intersects with psychiatric symptomatology? Where does the line between these phenomena get crossed? As a psychiatric resident and person who was raised in the Pentecostal faith, I have observed faith and psychopathology come to a head in the last 6 months. COVID-19 has dealt a religious strain of undocumented cases; I hope to shed light on the topic by sharing my experience of navigating the assessment and treatment plan of patients with psychiatric symptoms whose spiritual beliefs are a cornerstone of life.

\section{Piety, or pathology?}

The following approaches have helped me to identify what is driven by faith vs what is psychopathology:

While taking the patient history. Obtaining a history from a patient who professes to have strong spiritual beliefs and presents with psychiatric symptoms is similar to a standard patient interview, but pay special attention to how the patient came to the emergency department. Was there a family member, friend, or emergency medical services present at that time? During the

interview, patients often appear "normal," which may lead a clinician to question the reason for the consult, yet considering the recent events preceding the presentation will be a good place to start gathering the appropriate information for investigation.

Next, compare the patient's recent daily functioning with his/her baseline. If this information comes solely from the patient, it may be skewed, so try to retrieve information from a collateral source. If the patient was accompanied by someone, request permission from the patient to speak with him/her. It may also be best in some instances to speak with the collateral source out of earshot of the patient. Be aware that collateral information that comes from just one source also could be biased, so search for additional contacts to help acquire a comprehensive representation of the circumstances.

Information about a patient could come from a faith leader because people often rely on their faith leaders when they are ill, in need of support, or in crisis. ${ }^{2}$ Faith leaders may have valuable information and insight into the patient and the history of the patient's illness. In addition, diverse sources of collateral reports may be helpful because specific spiritual views and

LET YOUR VOICE BE HEARD

CURRENT PSYCHIATRY invites psychiatry residents to share their views on professional or clinical topics for publication in Residents' Voices. E-mail jbauer@mdedge.com for author guidelines.

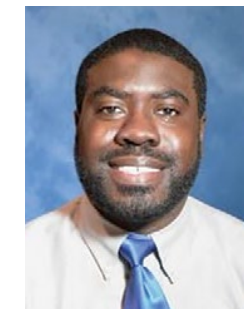

Dr. Barrett is a PGY-IV Psychiatry Resident and Co-Chief Resident, Department of Psychiatry and Behavioral Medicine, Charleston Area Medical Center, West Virginia University, Charleston, West Virginia.

\section{Disclosure}

The author reports no financial relationships with any companies whose products are mentioned in this article, or with manufacturers of competing products.

\section{Acknowledgment}

The author wishes to acknowledge the mentorship of Dr. Kerr, Associate Professor, Department of Psychiatry and Behavioral Medicine, Charleston Area Medical Center, West Virginia University, Charleston, West Virginia. doi: 10.12788/cp.0072 


\section{Clinical Point}

\section{Be sensitive to the patient's spiritual practices and receptive to learning about unfamiliar spiritual beliefs}

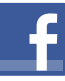

Discuss this article at www.facebook.com/ MDedgePsychiatry practices can vary even within one family or congregation. What may be an abnormal practice to some followers may be normal for others. ${ }^{3}$ When approaching these situations with parishioners, it is essential to maintain confidentiality.

While performing the clinical examination. As with any psychiatric diagnosis, other causative factors (metabolic and organic) need to be ruled out. Also, assess for the use of mood-altering substances. The patient may express offense or resistance to such questions, but maintain a matter-of-fact approach and explain that assessment for substance use is a routine part of the clinical examination. Approximately $18 \%$ of people in the United States with psychiatric disorders have a comorbid substance use disorder. ${ }^{4}$ However, keep in mind that a patient who refuses substance use screening is not necessarily hiding something. The road to being thorough may lead to strained rapport with the patient, and this risk must be balanced with providing the best care. As in any other clinical situation, seek evidence to both verify and clarify information without being deterred by a patient's vocalization of spiritual tenants.

\section{Learn about your patients' beliefs}

Do not feel defeated if you find these interviews difficult. Religion and symptomatology can overlap and fluctuate within the same faith group, which can make these types of assessments complex. ${ }^{5}$ In an effort to understand the patient more clearly, be sensitive to their spiritual practices and receptive to learning about unfamiliar spiritual beliefs. Be transparent about not knowing a specific belief or practice, and exhibit humility. Most patients are open to sharing their religious/spiritual views with a clinician who is sincere about wanting insight. Understanding the value of spiritual care is an important skill that many medical practitioners often lack. ${ }^{6}$ This understanding is especially critical when patients express worries related to the COVID-19 pandemic and how they are coping.

\section{Integrate your patient's spiritual requests}

If you are comfortable with certain practices that do not compromise your values or beliefs or put a patient at risk, try to integrate your patients' spiritual request(s) in their care. For a patient who serves a higher power, admitting to a problem (eg, fears related to COVID-19) or seeking professional help for symptoms (eg, anxiety, depression) may imply spiritual doubt. Patients may believe that seeking professional assistance means they are questioning the omnipotence of their deity to prevent or heal a condition. While spiritual distress can stimulate changes in behavior, it may not be pathological.

To avoid misdiagnosis, refer to the description "V62.89 (Z65.8) Religious or Spiritual Problem" in the DSM-5.7 If you find that it is a discord in faith that is affecting the patient's presentation, and that this has not caused a psychiatric disorder, document this appropriately and provide the necessary resources to continue supporting the patient holistically.

\section{References}

1. Dein S, Loewenthal K, Lewis CA, et al. COVID-19, mental health and religion: an agenda for future research. Mental Health, Religion \& Culture. 2020;23(1):1-9.

2. American Psychiatric Association Foundation. Mental health: a guide for faith leaders. Arlington, VA: American Psychiatric Association Foundation; 2018.

3. Johnson CV, Friedman HL. Enlightened or delusional? Differentiating religious, spiritual, and transpersonal experiences from psychopathology. Journal of Humanistic Psychology. 2008;48(4):505-527.

4. Han B, Compton WM, Blanco C, et al. Prevalence, treatment, and unmet treatment needs of US adults with mental health and substance use disorders. Health Aff (Millwood). 2017;36(10):1739-1747.

5. Menezes Jr A, Moreira-Almeida A. Differential diagnosis between spiritual experiences and mental disorders of religious content. Rev Psiq Clín. 2009:36(2):75-82.

6. Best M, Butow P, Olver I. Doctors discussing religion and spirituality: a systematic literature review. Palliat Med. 2016;30(4):327-337.

7. Diagnostic and statistical manual of mental disorders, 5 th ed. Washington, DC: American Psychiatric Association; 2013:725. 